\section{Bacterial glutathione import}

Glutathione (GSH) is essential across all kingdoms of life, playing critical roles in cysteine metabolism, protection against cell stress, control of cellular redox levels and facilitation of various signaling pathways. Gram-negative bacteria use a dedicated

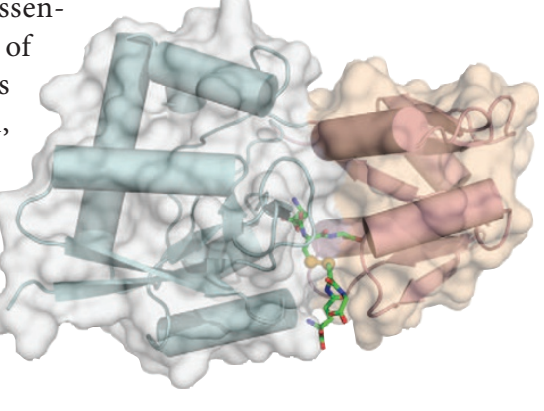
$\mathrm{ABC}$ transport system to import

GSH. Understanding how GSH accumulates in Gram-positive bacteria has come primarily from studies of Streptococcus mutans, commonly found in dental plaque. S. mutans can make its own GSH, but it also imports GSH to create intracellular concentrations v25 times higher than those attained from de novo synthesis. Despite the importance of GSH, there is limited information on the cellular machinery required for its import in Gram-positive bacteria. To address this, Vergauwen, Savvides and colleagues have investigated the function of SMU_1942c from S. mutans (referred to as GshT). Previous work revealed that this protein was required for GSH assimilation. Now, the authors find that GshT preferentially binds GSH and also displays affinity for GSH variants. The crystal structure of GshT in complex with glutathione disulfide (GSSG) reveals a bilobed structure with the ligand-binding site lying in the deep cleft between the two lobes. As with other solute-binding proteins, GSSGbound GshT is in a closed conformation and probably undergoes a Venus flytrap-like conformational change when it binds ligand. Close inspection of the binding pocket explains how GshT can bind a variety of GSH derivatives. The protein makes intimate contacts with the first glutamyl moiety of GSSG, reflecting how it would capture GSH, while forming only a few specific interactions with the second. Additional studies using a gsht mutant confirm that GshT is responsible for GSH import in S. mutans. The authors further show that GshT primes import through TcyABC, an $\mathrm{ABC}$ transporter previously associated with import of $\mathrm{L}$-cystine, a source of intracellular cysteine. This dual import pathway may become an attractive target for controlling S. mutans virulence. (Mol. Microbiol. http://dx.doi.org/10.1111/ mmi.12274, published online 23 May 2013)

$M M$

\section{Sensing KATastrophe}

How DNA double-strand breaks (DSBs) are sensed and signaled to promote checkpoint activation and DNA repair has remained elusive. In mammalian cells, the checkpoint kinase ATM is recruited to DSBs through association with the Mre11-Rad50-Nbs1 complex and activates downstream components of the DNA-damage response (DDR). Now, work by Kaidi and Jackson reveals how damageinduced chromatin modifications regulate ATM activity. They show that the lysine acetyltransferase KAT5, previously known to acetylate and stimulate ATM, is itself phosphorylated in cells exposed to ionizing radiation. The modified residue, Tyr44, is within the chromodomain that mediates KAT5 interaction with trimethylated histone H3 Lys9 (H3K9me3) and other chromatin marks, and its phosphorylation enhances both chromatin binding affinity and ATM acetylation activity. $\mathrm{H} 3 \mathrm{~K} 9 \mathrm{me} 3$ peptides stimulate ATM acetylation by KAT5 in vitro, and Tyr44 mutations that prevent phosphorylation impair ATM-induced checkpoint activation in vivo, rendering cells hypersensitive to ionizing radiation. In contrast, Tyr44 mutation does not affect KAT5's transcriptional activation functions, thus suggesting that Tyr44 phosphorylation is required for DDR signaling. The role of chromatin structure in KAT5-mediated ATM induction is examined by monitoring the effect of increasing cellular histone acetylation levels by TSA treatment or by HP1 depletion to expose H3K9me3. Remarkably, both conditions induce Tyr44 phosphorylation and KAT5 chromatin retention to promote ATM acetylation and phosphorylation of ATM substrates in the absence of DSBs. These observations suggest that KAT5 and ATM may also function in a genome surveillance pathway signaling repair in response to chromatin alterations. Lastly, inhibition or depletion of the tyrosine kinase $\mathrm{c}$-Abl abolishes both KAT5 Tyr44 phosphorylation and ATM-dependent cell-cycle arrest, thus explaining previous observations linking $\mathrm{c}-\mathrm{Abl}$ and the ionizing radiation-induced checkpoint response. This work provides crucial molecular insights into how chromatin changes are read and interpreted by cellular signaling pathways. (Nature 498, 70-74, 2013)

$B M$

\section{SAS, refined}

Small-angle scattering (SAS) is a technique that provides thermodynamic and structural information about macromolecules in solution, such as particle size and mass distribution. Notwithstanding the one-dimensional nature of its data, SAS is sensitive to perturbations in experimental conditions, and this hampers extraction of information from the SAS profile. With the lack of widely accepted standards for the assessment of SAS data analysis and over-fitting, SAS has remained a complementary technique with limited utility in cases where the macromolecule is flexible, for which key parameters often cannot be determined. Now Rambo and Tainer describe new approaches to address these fundamental SAS limitations. They discovered that an empirically determined scattering ratio, termed the volume of correlation $\left(V_{c}\right)$, greatly facilitates accurate measurement of particle mass, even in cases of conformational flexibility. The $V_{c}$ parameter can be used to calculate a new residual, $R_{\mathrm{SAS}}$, which describes the extent of agreement between a structural model and experimental data. Tapping principles from information theory, the authors also developed an approach to calculate a cross-validated statistical indicator of over-fitting, $\chi_{\text {free }}^{2}$, analogous to the $R_{\text {free }}$ statistic in crystallography. Models with minimal $R_{\text {SAS }}$ values constrained by a fixed $\chi^{2}$ free limit are unlikely to be over-fitted in high-noise datasets, and the $\chi^{2}$ free cutoff provides a meaningful, objective definition of SAS resolution limit. These developments extend the power of SAS to a wider range of macromolecules than was previously possible and add much-needed rigor to the analysis and interpretation of SAS data. (Nature 496, 477-481, 2013) 